\title{
Quantitative Elemental Mapping of Alkali Activated Slag Cement
}

Najat A. Alharbi ${ }^{1}$, Richard K. Hailstone ${ }^{1}$, and Benjamin Varela ${ }^{2}$

1. Center for Imaging Science, Rochester Institute of Technology, 54 Lomb Memorial Dr, Rochester, NY 14623, USA

2. Department of Mechanical Engineering Rochester Institute of Technology, 76 Lomb Memorial Dr, Rochester NY 14623, USA

Blast furnace slag is a non-metallic byproduct generated by the production of iron and steel in a blast furnace at temperatures in the range of $1400^{\circ}-1600^{\circ} \mathrm{C}$. The alkali activation of blast furnace slag has the potential to reduce the environmental impact of cementitious materials and to be applied in geographic zones where weather is a factor that negatively affects performance of materials based on Ordinary Portland Cement. Alkali-activated blast furnace slag cements have been studied since the 1930s due to its high compressive strength; they can exceed $100 \mathrm{MPa}$ in 28 days. The low $\mathrm{Ca} / \mathrm{Si}$ ratio in slag improves its resistance to aggressive chemical materials such as acids, chlorides and sulphates [1,2, 3].

Blast furnace slag is a highly heterogeneous material. It is well known that its chemical composition affects the physical properties of the alkali activated material, however there is little work on how these inhomogeneities affect the microstructure and pore formation. In this study we characterize slag cement activated with $\mathrm{KOH}$ using scanning electron microscopy (SEM), x-ray microanalysis and quantitative element mapping. Attention is focused on delineating the phases [4] induced by the alkali activation, as these phases are important in determining the mechanical properties of the material. Quantitative elemental mapping was done with Bruker Qmap software on spectrum images using standard-based elemental quantification to show the quantitative spatial distribution of each element of interest.

Figure 1 shows the BSE image of the cement sample (top left) and quantitative mapping images for the elements magnesium, silicon, potassium, calcium, and aluminum. The BSE image is divided into "particles" (bright features) and "background" (nonbright features). Magnesium and aluminum show a uniform distribution over the particles areas while potassium appears only in the background areas that correspond to the formation of calcium aluminosilicate (C-A-S-H) where aluminum substitutes for silicon is possibly formed. Silicon and calcium show a relatively high concentration in the particles with concentration between 14-20 for Si and 17-25 for $\mathrm{Ca}$, with much lower concentration in the background.

\section{References}

[1] Hua et al. Characterization of aged slag concretes. ACI Materials Journal, 105 (2) (2008).

[2] Shi, C., Jiménez, A. F., \& Palomo, A. New cements for the 21st century: the pursuit of an alternative to Portland cement. Cement and Concrete Research, 41 (7), 750-763 (2011).

[3] Rackel et al, Distinctive microstructural features of aged sodium silicate-activated slag concretes. Cement and Concrete Research, 65, 41-51 (2014).

[4] Alharbi, Najat, Hailstone, Richard, Varela, Benjamin. EDS-Based Phase Analysis of Alkali Activated Slag. Microscopy \& Microanalysis 2017 Meeting. St. Louis, Missouri. 

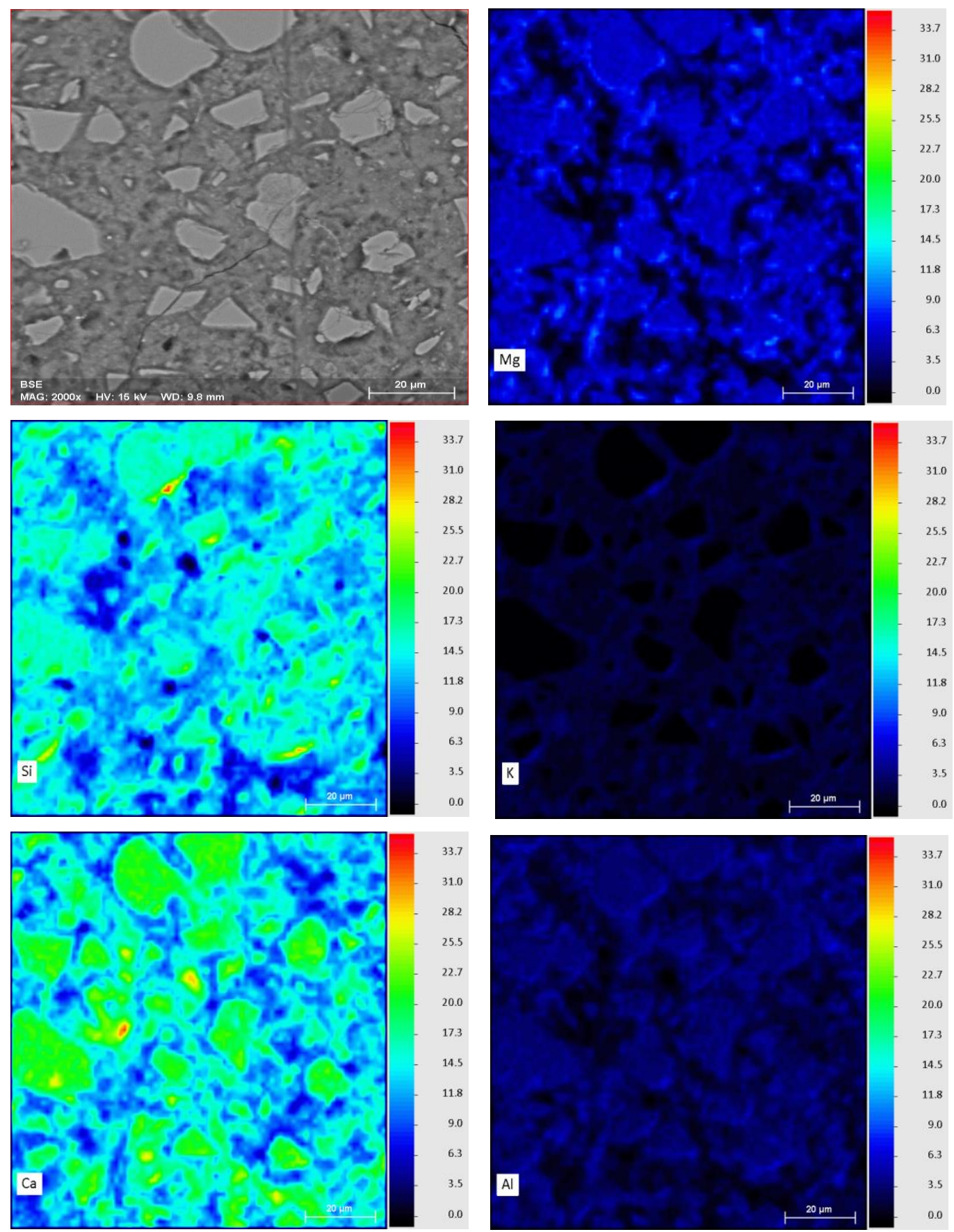

Figure 1. BSE image of the alkali activated slag (top left), and quantitative mapping of the elements. Color scale in atom\%. 